\title{
Void growth and damage ahead of a crack in pressure-sensitive dilatant polymers
}

\author{
H. B. Chew, T. F. Guo \& L. Cheng \\ Department of Mechanical Engineering, \\ National University of Singapore, Singapore
}

\begin{abstract}
The deformation of amorphous polymers is highly sensitive to hydrostatic pressure and the plastic flow is non-volume preserving. Failure of these materials is often preceded by the formation of small crack-like defects known as crazes, bridged by the fibrils of the polymer. In this work, a population of discrete voids is placed ahead of a crack within a boundary layer configuration to study the effects of pressure-sensitivity $\alpha$ and plastic dilatancy $\beta$ on void growth and damage in polymeric materials. Results show that high pressure-sensitivity significantly increases both the intensity and spatial extent of damage, which could promote crazing. These effects can be compounded as the deviation from the associated flow rule, $|\beta-\alpha|$, increases. This study offers some evidence of pressure-sensitivity and plastic dilatancy as the major contributing parameters to the formation and growth of microporous crazing zones in polymers.
\end{abstract}

Keywords: void growth, coalescence, pressure-sensitive yielding, plastic dilatancy, polymer.

\section{Introduction}

The ductile fracture of many engineering materials involves the micromechanical process of void growth and coalescence. The fracture surfaces of these materials contain voids of various size-scales. In polymeric materials, the larger voids can originate from cavitated rubber blends or from decohesion of filler particle/polymer matrix interfaces, while shear banding and crazing can induce the formation of the smaller microvoids. The ductile fracture process can be described by mechanism-based computational approaches which employ voidcontaining cell elements [1]. Porous material models, such as the Gurson 
constitutive model [2], are used to describe void growth for each cell element, and the macroscopic softening during the fracture process. This approach has been widely used in the prediction of ductile crack growth in metals. More recently, Guo and Cheng [3] extended the Gurson model to incorporate vapour pressure effects. The extended Gurson model has been used to study the vapour pressure assisted void growth and rupture of hygroscopic polymeric materials, like the die attach and molding compound in electronic packages [4-6].

While the micromechanisms of ductile fracture for metals and polymers are somewhat similar, two important characteristics of polymers that differ from metals are its pressure-sensitive yielding, and its non-volume preserving plastic flow. To this end, Chew et al. [7] examined the effects of microvoid interaction in pressure-sensitive dilatant polymeric materials. They showed that increasing pressure-sensitivity severely reduces the material's stress carrying capacity, while multiple void interactions was responsible for the sharp post-peak stress drop and could act as a catalyst for rapid failure. In addition, plastic dilatancy was found to have some effect in raising the post-peak stress levels, resulting in a larger work of separation.

An accurate simulation of the void growth behaviour in pressure-sensitive dilatant polymeric materials can be achieved by explicitly modelling the voids using refined finite elements. This discrete void approach was previously adopted by Tvergaard and Hutchinson [8] and Kim et al. [9] for the study of the ductile fracture of metals, and by Chew et al. [10] for the modelling of moisture induced failure of hygroscopic polymeric materials.

In this work, a single row of discrete voids is placed ahead of a crack within a boundary layer configuration to study void growth and damage in pressuresensitive dilatant polymeric materials (Fig. 1). The pressure-sensitive yielding of the material is described by a linear combination of the mean stress and effective stress under a non-associated flow rule. The motivation behind this study is to understand the contributions of pressure-sensitivity $\alpha$ and plastic dilatancy $\beta$ to the formation and growth of long craze zones widely observed in polymeric materials [11].
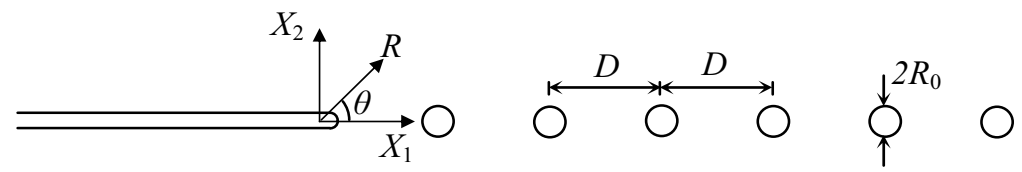

Figure 1: Schematic of cylindrical discrete voids ahead of a crack in a boundary layer configuration.

\section{Problem formulation}

\subsection{Material model}

The plastic behaviour of polymeric materials differs considerably from the von Mises material. Such behaviour can be explained by assuming a yield criterion 
based on a linear combination of the mean stress and effective stress. Here, the pressure-dependent yielding of the material is described by

$$
\sqrt{3 J_{2}}+\sigma_{m} \tan \alpha-\hat{\sigma}=0
$$

where $J_{2}$ is the second invariant of the deviatoric part of the Cauchy stress tensor $\boldsymbol{\sigma}, \sigma_{\mathrm{m}}$ the mean stress, $\hat{\sigma}$ the flow stress of the subsequent yield surface, and $\alpha$ the index for pressure-sensitivity. We assume the flow potential to take the form

$$
\Phi=\sqrt{3 J_{2}}+\sigma_{m} \tan \beta
$$

where $\beta$ is the index for plastic dilatancy. The plastic deformation rate is given by the non-associated flow rule

$$
\mathbf{d}^{\mathrm{P}}=\dot{\varepsilon}^{\mathrm{P}} \frac{\partial \Phi}{\partial \boldsymbol{\sigma}}
$$

where $\dot{\varepsilon}^{\text {P }}$ is the equivalent strain rate.

The flow stress $\hat{\sigma}$ is a function of the accumulated plastic strain $\varepsilon^{\mathrm{P}}=\int \dot{\varepsilon}^{\mathrm{P}} d t$. For a power-law plastic hardening solid, one has

$$
\frac{\sigma_{0}}{E}\left(\frac{\hat{\sigma}}{\sigma_{0}}\right)^{1 / N}-\frac{\hat{\sigma}}{E}=\varepsilon^{\mathrm{P}}
$$

where $N$ is the hardening exponent ranging from 0 to 1 , and $\sigma_{0}$ the initial yield stress under pure shear which is related to the initial tensile and compressive yield stresses $\sigma_{0}{ }^{\mathrm{t}}$ and $\sigma_{0}{ }^{\mathrm{c}}$, by

$$
\sigma_{0}= \begin{cases}\left(1+\frac{1}{3} \tan \alpha\right) \sigma_{0}^{\mathrm{t}} & \text { for tension } \\ \left(1-\frac{1}{3} \tan \alpha\right) \sigma_{0}^{\mathrm{t}} & \text { for compression. }\end{cases}
$$

The pressure-sensitivity index $\alpha$ can be determined from $\sigma_{0}{ }^{\mathrm{t}}$ and $\sigma_{0}{ }^{\mathrm{c}}$ as

$$
\alpha=\tan ^{-1}\left(3 \frac{\sigma_{0}^{\mathrm{c}}-\sigma_{0}^{\mathrm{t}}}{\sigma_{0}^{\mathrm{c}}+\sigma_{0}^{\mathrm{t}}}\right) .
$$

In polymeric materials, typical pressure-sensitivity levels range between $0^{\circ}$ to $23^{\circ}$.

\subsection{Small-scale yielding}

Xia and Shih [1] simplified the ductile fracture process under small-scale yielding conditions by placing a single row of void-containing cells ahead of the crack. In the present study, we model void growth and damage via an array of unit-cells ahead of the crack (see Fig. 1). Each unit-cell is of dimensions $D$ by $D$ and contains a discrete cylindrical void of initial radius $R_{0}$. The initial void volume fraction is given by $f_{0}=\pi R_{0}^{2} / D^{2}$. A total of 23 voids are used in the computation. 
(a)

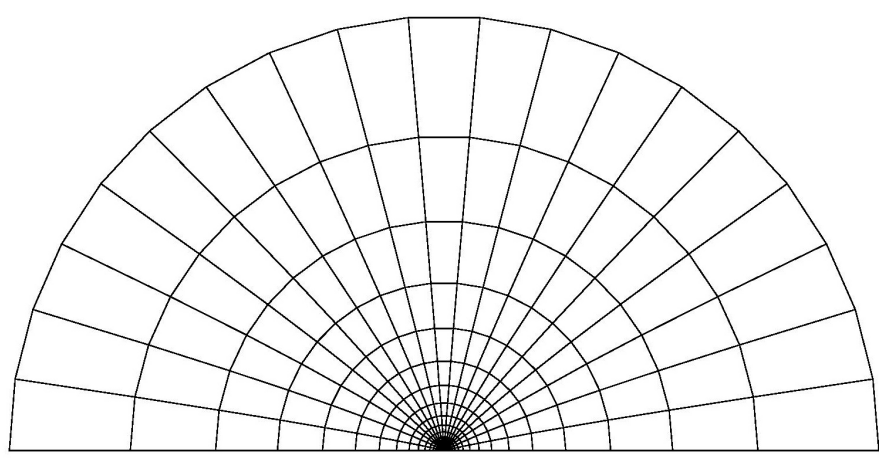

(b)

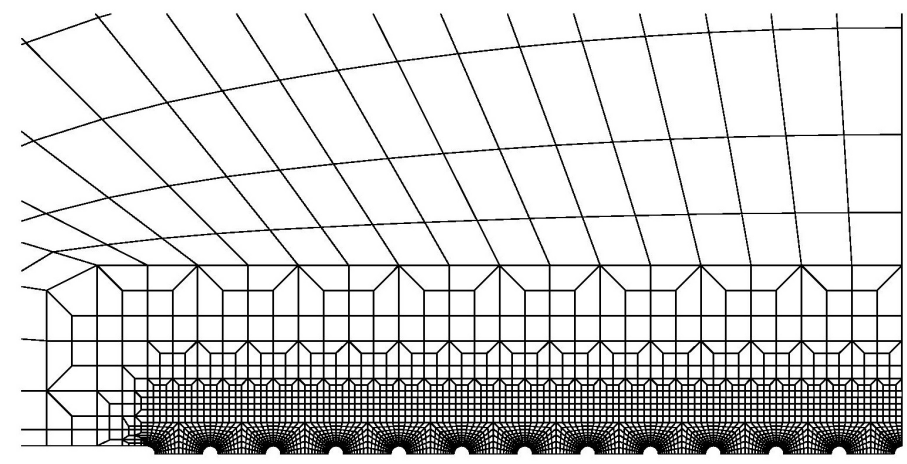

Figure 2: $\quad$ Finite element mesh for small-scale yielding analysis. (a) Entire mesh showing remote boundary. (b) Close-up view of the crack tip.

Along the remote circular boundary (see Fig. 2a), the elastic asymptotic (inplane) displacement field

$$
\begin{aligned}
& u_{1}(R, \theta)=K_{I} \frac{1+v}{E} \sqrt{\frac{R}{2 \pi}}(3-4 v-\cos \theta) \cos \frac{\theta}{2} \\
& u_{2}(R, \theta)=K_{I} \frac{1+v}{E} \sqrt{\frac{R}{2 \pi}}(3-4 v-\cos \theta) \sin \frac{\theta}{2}
\end{aligned}
$$

is applied under plane strain conditions, where $R^{2}=X_{1}^{2}+X_{2}^{2}$ and $\theta=\tan ^{-1}\left(X_{2} / X_{1}\right)$ for points on the remote boundary. $K_{\mathrm{I}}$ is the mode I stress intensity factor related to the $J$-integral by

$$
J=\frac{1-v^{2}}{E} K_{I}^{2} .
$$

At various stages of loading, the value of the $J$-integral is calculated on a number of contours around the crack using the domain integral method. The domain integral value was found to be in good agreement with the value given by eqn. (8) for the prescribed amplitude $K_{\mathrm{I}}$. This consistency check assures that smallscale yielding conditions are satisfied. 
Figure 2 displays an example finite element mesh for the discrete void model. By taking advantage of symmetry, only one half of the geometry needs to be modelled. The mesh contains 4-node bilinear quadrilateral hybrid plane strain elements. The computations are performed within the finite strain setting using the general-purpose finite element program ABAQUS Version 6.5.1 [12].

\section{Crack tip plastic zone}

Before proceeding to the modeling of void growth, we first examine the effects of pressure-sensitivity $\alpha$ on the extent of plastic deformation ahead of the crack. Some insights can be obtained from the Dugdale strip yield model, which assumes a long slender plastic zone at the crack tip in a nonhardening pressureinsensitive material $\left(\alpha=0^{\circ}\right)$. The plastic zone size $L$ under a uniaxial tensile stress $\sigma_{\mathrm{T}}$ is given as

$$
L=\frac{\pi}{8}\left(\frac{K_{I}}{\sigma_{T}}\right)^{2} .
$$

For the pressure-sensitive material in eqn. (1), the tensile stress $\sigma_{\mathrm{T}}$ at yielding is $\sigma_{\mathrm{T}}=\sigma_{0} /(1+1 / 3 \tan \alpha)$. Denoting $L_{0}$ as the plastic zone size corresponding to $\alpha=$ $0^{\circ}$, i.e. $\sigma_{\mathrm{T}}=\sigma_{0}$, we obtain

$$
L / L_{0}=\left(1+\frac{1}{3} \tan \alpha\right)^{2}
$$

This relation suggests an increase in the plastic zone size $L$ with pressuresensitivity $\alpha$. Observe that an increase in $\alpha$ from $0^{\circ}$ to $20^{\circ}$ increases $L$ by around $25 \%$.

A numerical study is next performed to shed some light on the extent of plastic dissipation in a void-free pressure-sensitive polymer. The void-free material is subjected to mode I, $K$-field loading in eqn. (7). An associated flow is assumed with $\alpha=\beta$. The elastic modulus of the polymer is taken to be $E=500$ $\mathrm{MPa}$, with a yield strength of $\sigma_{0}=5 \mathrm{MPa}$, and a Poisson's ratio of $v=0.4$. In accordance with the Dugdale assumption, the strain hardening exponent $N$ is set to zero (for ideally-plastic material).

Figure 3 displays the plastic zones in the deformed mesh configuration for three pressure-sensitive levels: $\alpha=0^{\circ}, 10^{\circ}$ and $20^{\circ}$. The plastic zones are operationally defined by $\varepsilon^{\mathrm{p}} \geq 0.001$, where $\varepsilon^{\mathrm{p}}$ is the accumulated plastic strain. Observe that at the same external load level, the plastic zone spreads out as the pressure-sensitivity level increases. For example, at $J=68 \mathrm{kNm}^{-1}$, an increase in $\alpha$ from $0^{\circ}$ to $20^{\circ}$ raises the extent of plastic dissipation ahead of the crack by nearly two-fold $\left(L / L_{0} \approx 2\right)$. This general trend of increasing plastic dissipation with pressure-sensitivity corroborates with the findings from eqn. (10).

\section{Damage evolution in porous polymers}

In this section, an array of discrete voids is placed ahead of the crack. From dimensional considerations, the spatial distribution of the current void volume 
fraction $f$ depends on the following dimensionless geometric-material parameters:

$$
\frac{J}{\sigma_{0} D} ; \frac{\sigma_{0}}{E}, N, v, \alpha, \beta ; f_{0} .
$$

The properties of the polymeric material are specified by the parameters $\sigma_{0} / E=$ $0.01, v=0.4$, and $N=0.1$. We focus on the effects of pressure-sensitivity and plastic dilatancy on void growth and damage ahead of the crack.

(a)

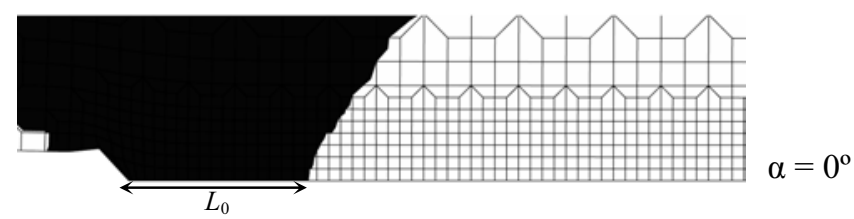

(b)

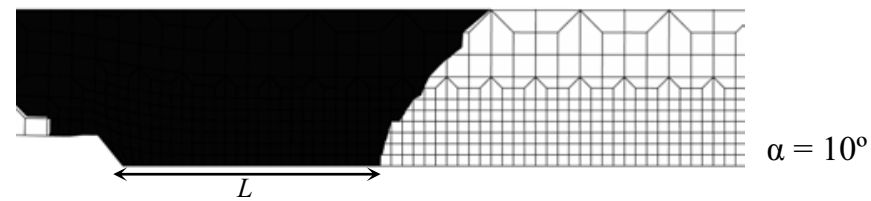

(c)

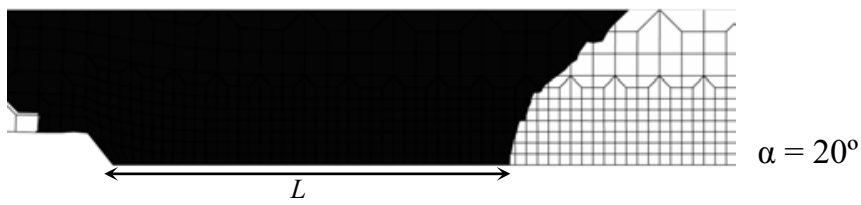

Figure 3: Plastic zones in the deformed mesh configuration for several pressure-sensitivity levels at $J=68 \mathrm{kNm}^{-1}$.

\subsection{Pressure-sensitivity effects}

The effects of pressure-sensitivity under an associated flow rule $(\alpha=\beta)$ are shown in Fig. 4 for $f_{0}=0.005$ and $f_{0}=0.05$. Computations focusing on the ductile fracture of metals by Tverggard and Hutchinson [8] have identified two distinct mechanisms of fracture: (i) single void growth mechanism, and (ii) multiple void interaction mechanism. Both these failure mechanisms are also exhibited in our study for amorphous polymers.

At equivalent load levels, we observe that an increase in $\alpha$ significantly increases both the intensity and extent of damage ahead of the crack. Herein, we operationally define the spatial extent of damage as the distance from the crack tip to the region where the void porosity has increased by $f-f_{0}>0.01$. For $f_{0}=$ 0.005 at $J /\left(\sigma_{0} D\right)=0.36$, an increase in $\alpha$ from $0^{\circ}$ to $15^{\circ}$ raises the peak porosity level by $160 \%$ and increases the extent of damage by nearly three-fold. Similar observations are made for $f_{0}=0.05$.

The plastic zone distribution in the deformed mesh configuration for $f_{0}=$ 0.005 is displayed in Fig. 5. Observe that the plastic zone spreads over a much larger region as $\alpha$ increases. For $\alpha=0^{\circ}$, the plastic zone is confined to the first 
two voids ahead of the crack, resulting in near-tip void growth. For $\alpha=15^{\circ}$, the plastic zone spreads across the first four voids, leading to the formation of an extended damage zone. This extended damage zone closely resembles the long craze zones in polymers. One can therefore infer that pressure-sensitivity is a major contributing parameter to the crazing phenomenon. Interestingly, we also note that there is an increasing tendency for the voids to become oblate as they grow larger. This suggests the effects of void shape to play an important role in the coalescence stages of fracture.
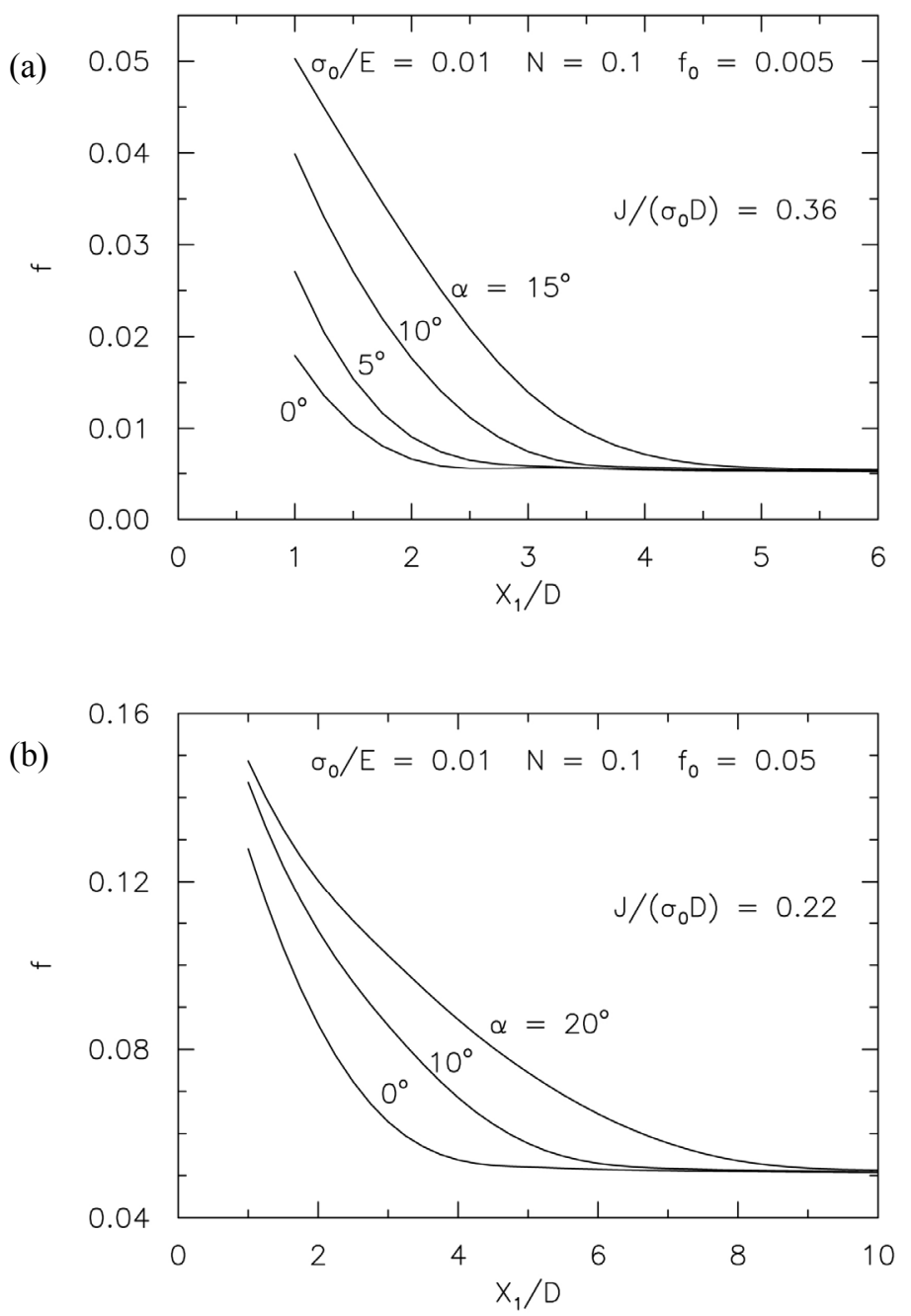

Figure 4: Distribution of porosity $f$ ahead of crack $\left(X_{2}=0\right)$ for several pressure-sensitivity levels with $\alpha=\beta$, (a) $f_{0}=0.005$; (b) $f_{0}=0.05$. 
(a)

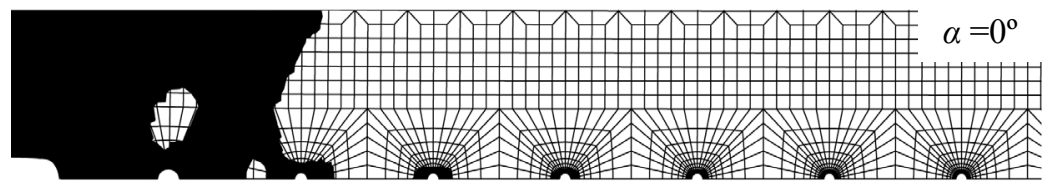

(b)

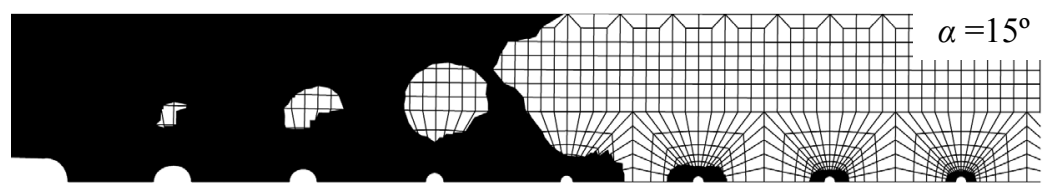

Figure 5: Plastic zones in the deformed mesh configuration for several pressure-sensitivity levels at $J /\left(\sigma_{0} D\right)=0.36$. $f_{0}=0.005$, (a) $\alpha=0^{\circ}$; (b) $\alpha=15^{\circ}$.

\subsection{Plastic-dilatancy effects}

The analysis in the previous section employs an associated flow rule, i.e. $\alpha=\beta$. Experimental studies, however, have shown that the plastic volume change in polymers does not commensurate with the predictions of the associated flow rule, i.e. $\beta<\alpha$.

Figure 6 displays the effects of $\beta$ on the damage distribution ahead of the crack for $\alpha=10^{\circ}$ and $20^{\circ}$. One can see that an increase in the deviation from the associated flow, $|\beta-\alpha|$, significantly increases both the intensity and the spatial extent of damage. For $f_{0}=0.005, \alpha=20^{\circ}$, at $J /\left(\sigma_{0} D\right)=0.60$, an increase in $|\beta-\alpha|$ from $0^{\circ}$ to $5^{\circ}$ raises the damage intensity level by nearly $60 \%$, and shifts the damage process zone from $X_{1}=5 D$ to $X_{1}=7 D$. The effects of increasing $|\beta-\alpha|$ are found to be less severe for $f_{0}=0.05$. The non-conservative nature of the associated flow in predicting damage has serious implications in the design of engineering materials and structures.

\section{Conclusion}

The effects of pressure-sensitivity, $\alpha$, and plastic dilatancy, $\beta$, on void growth and damage in amorphous polymeric materials have been studied. Our numerical results for a void-free polymeric material show that pressure-sensitivity significantly increases the extent of plastic dissipation ahead of the crack. Void growth and damage in the pressure-sensitive dilatant polymeric material was subsequently modelled via a single row of discrete voids placed ahead of the crack. Both the voiding intensity and the spatial extent of damage were observed to increase with pressure-sensitivity, which could promote crazing. These effects were further compounded as the deviation from the associated flow rule, $|\beta-\alpha|$, increases. These findings suggest pressure-sensitivity and plastic dilatancy to be closely linked with the formation of long craze zones in polymers. 
(a)

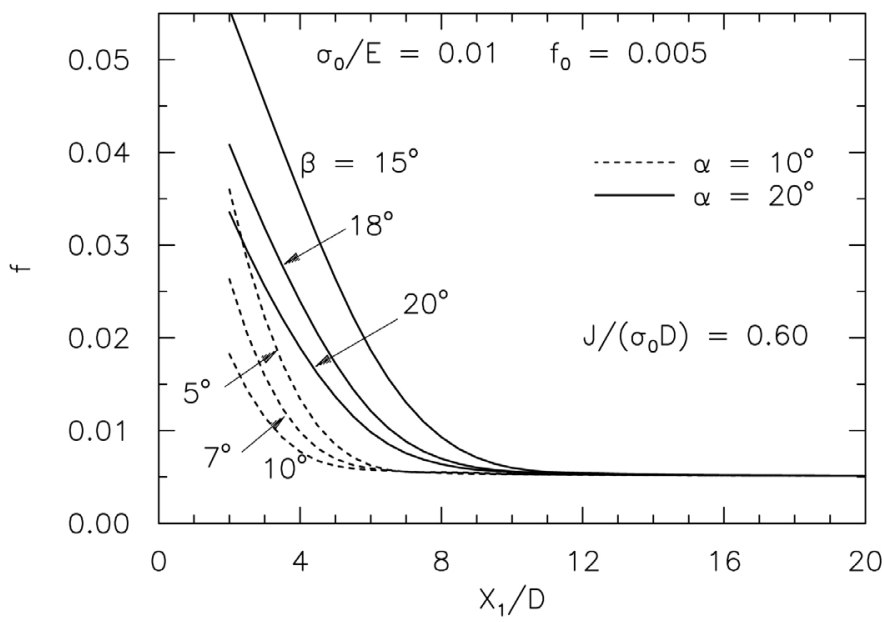

(b)

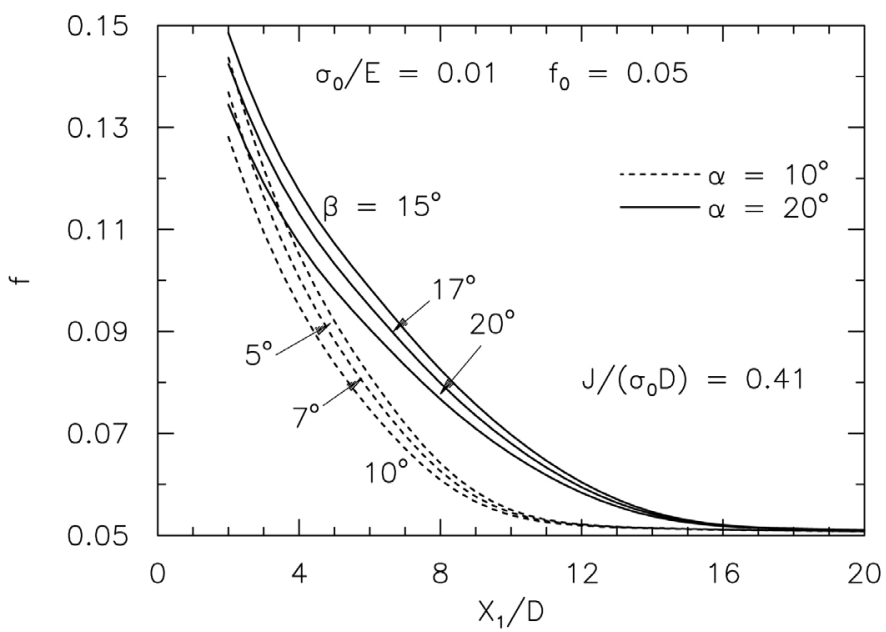

Figure 6: Distribution of porosity $f$ ahead of crack $\left(X_{2}=0\right)$ for several plastic dilatancy levels with $\alpha=10^{\circ}$ and $20^{\circ}$, (a) $f_{0}=0.005$; (b) $f_{0}=0.05$.

\section{References}

[1] Xia, L. \& Shih, C.F., Ductile crack growth - I. A numerical study using computational cells with microstructurally-based length scales. Journal of the Mechanics and Physics of Solids, 43, pp. 233-259, 1995.

[2] Gurson, A.L., Continuum theory of ductile rupture by void nucleation and growth: Part I - Yield criteria and flow rules for porous ductile media. Journal of Engineering Materials and Technology, 99, pp. 2-15, 1977. 
[3] Guo, T.F. \& Cheng, L., Modeling vapor pressure effects on void rupture and crack growth resistance. Acta Materialia, 50, pp. 3487-3500, 2002.

[4] Chew, H.B., Guo, T.F. \& Cheng, L., Vapor pressure and residual stress effects on the toughness of polymeric adhesive joints. Engineering Fracture Mechanics, 71, pp. 2435-2448, 2004.

[5] Chew, H.B., Guo, T.F. \& Cheng, L., Vapor pressure and residual stress effects on failure of an adhesive film. International Journal of Solids and Structures, 42, pp. 4795-4810, 2005.

[6] Chew, H.B., Guo, T.F. \& Cheng, L., Vapor pressure and residual stress effects on mixed mode toughness of an adhesive film. International Journal of Fracture, 134, pp. 349-368, 2005.

[7] Chew, H.B., Guo, T.F. \& Cheng, L., Effects of pressure-sensitivity and plastic dilatancy on void growth and interaction. International Journal of Solids and Structures, in Press, 2006.

[8] Tvergaard, V. \& Hutchinson, J.W., Two mechanisms of ductile fracture: void by void growth versus multiple void interaction. International Journal of Solids and Structures, 39, pp. 3581-3597, 2002.

[9] Kim, J., Gao, X. \& Srivatsan, T.S., Modeling of crack growth in ductile solids: a three-dimensional analysis. International Journal of Solids and Structures, 40, pp. 7357-7374, 2003.

[10] Chew, H.B., Guo, T.F. \& Cheng, L., Vapor pressure and voiding effects on thin film damage. Thin Solid Films, in Press, 2006.

[11] Kambour, R.P., A review of crazing and fracture in thermoplastics. Journal of Polymer Science, 7, pp. 1-154, 1973.

[12] Hibbit, Karlsson \& Sorensen Inc., Abaqus/Standard User's Manual, Version 6.5.1, Vol. 1, 2005. 